\title{
Determining the propagation path of a disturbance in multi-rate process and electromechanical systems
}

\author{
Inês M. Cecílio ${ }^{\mathrm{a}, 1, *}$, James R. Ottewill ${ }^{\mathrm{b}}$, Nina F. Thornhill ${ }^{\mathrm{a}}$ \\ ${ }^{a}$ Centre for Process System Engineering, Department of Chemical Engineering, Imperial \\ College London, London SW7 2AZ, UK \\ ${ }^{b}$ ABB Corporate Research Center, ul. Starowiślna 13a, 31-038 Kraków, Poland
}

\begin{abstract}
This paper proposes a multi-rate method to identify the propagation path of a persistent disturbance in an enlarged system envelope which includes the process plant and its electromechanical equipment. The need to integrate process and equipment diagnosis has been highlighted by industrial commentators. However, process and electromechanical measurements often have different sampling rates. The multi-rate method proposed extends a state-of-the-art propagation path method so that it combines fast-sampled electromechanical measurements and slow-sampled process measurements. The method is based on non-linear mutual prediction, which yields the directionality in the relationship between two time series. The method was demonstrated and validated, giving the expected outcome in an experimental case study, in which the root cause and propagation path of the disturbance were known.
\end{abstract}

Keywords: Fault diagnosis, root cause analysis, propagation, nonlinear time series analysis, multirate, chemical industry, machinery

\section{Introduction}

In chemical process plants, when a disturbance originates at the root cause, it often propagates through mass and energy flows, and control signals, thus

\footnotetext{
* Corresponding author

Email address: i.cecilio09@imperial.ac.uk (Inês M. Cecílio)

${ }^{1}$ Present address: Schlumberger Gould Research, High Cross, Madingley Road, Cambridge CB3 0EL
} 
affecting measurements in multiple parts of the plant (Thornhill and Horch, 2007). A common goal in process monitoring and diagnosis is to distinguish the root cause from the propagated disturbances. To that end, a recent topic in the literature is the extraction of the propagation path of the disturbance from measurement data (Yang and Xiao, 2012). The propagation path is a qualitative model of the affected system, and shows the affected measurements in a directed succession according to the order of propagation of the disturbance. Deriving the propagation path allows the root cause to be inferred by tracking the disturbance up the path.

Recently, there have been efforts to integrate process monitoring with the condition monitoring of the equipment and utilities which service the process, in particular to electromechanical equipment (Lindholm et al., 2011; Cecílio et al., 2011; Cecílio et al., 2014). The need for this integration has already been highlighted by several industrial commentators (Reeves, 2005; Schiltz, 2008). The reason is that these auxiliary subsystems interact with the process through energy and signal paths, and hence disturbances can propagate across the subsystems. The aim of this paper is to enable analyses of propagation path in an enlarged system envelope which includes the process and its electromechanical equipment.

Several methods to derive the propagation path of persistent disturbances have been successfully used in operations data of process systems, and some are available in commercial tools (Horch et al., 2007). These methods use advanced signal analysis in order to search for features that arise in the data when a disturbance propagates along a system. Examples of such features include time delays, attenuation, transfer of information, and conditional probability relations. Examples of methods include the quantification of the nonlinearity of time series (Thornhill, 2005), the transfer entropy between two time series (Bauer et al., 2007a; Naghoosi et al., 2013), and the non-linear mutual prediction between two time series (Bauer et al., 2007b; Stockmann et al., 2012).

However, the current methods are applicable only to uni-rate systems, that is, systems whose measurements are all available with the same sampling rate. 
Systems with process and electromechanical measurements, on the other hand, are often multi-rate because process measurements are usually sampled approximately 1000 times slower than electromechanical measurements. Therefore, to apply the current methods, the electromechanical measurements have to be downsampled to the process rate. However, downsampling may compromise the accuracy of the results. For instance, if the duration of the disturbance is shorter in the electromechanical measurements than in the process measurements, the slow process sampling rate may be enough to capture the disturbance in the process measurements but not in the electromechanical measurements. Such data sets would require the combined analysis of fast-sampled electromechanical measurements with slow-sampled process measurements.

The contribution of this paper is the adaptation of method by Bauer et al. (2007b) for the determination of the propagation path of a persistent disturbance. The adaptation enables the combined analysis of electromechanical and process measurements. The paper uses experimental data from a gas compressor rig to benchmark the results yielded with the new multi-rate method with the results of the uni-rate method by Bauer et al. (2007b).

The paper is structured as follows. Section 2 presents the experimental case study and underlying physical models for validating the method. Section 3 provides background on non-linear mutual prediction. Section 4 explains the algorithm for the multi-rate method, which is then tested and compared to the uni-rate method by Bauer et al. (2007b) in section 5. Section 6 closes with conclusions.

\section{Compressor rig experiments and physical modelling}

\subsection{Compressor rig experimental case study}

To validate the proposed method, the paper uses a case study in which the root cause of the disturbance is known and the expected propagation path is derived from a model of the system. The case study consists of measurement data from experimental work with a gas compressor rig located at ABB Corporate 


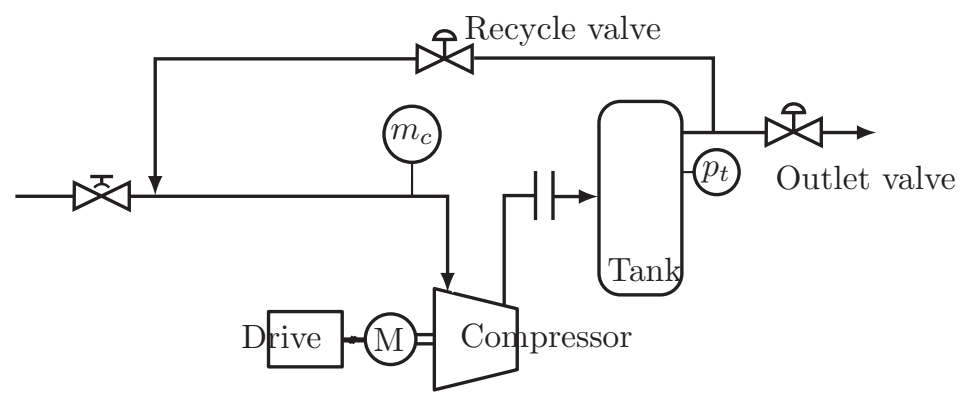

Figure 1: Simplified schematic of the gas compression rig.

Research Center, Kraków, Poland. The main components of the rig are a compressor, an induction motor and an a.c. voltage-source inverter drive. Figure 1 shows the rig schematically. On the process side, the measured variables relevant to this paper are the tank pressure, $p_{t}$, and the flow through the compressor, $m_{c}$. The electromechanical variables are measured in the drive and include the shaft speed set-point, $\omega^{*}$, the shaft speed, $\omega$, and the electromagnetic torque in the motor, $\tau_{e}$.

Figure 2 shows time series of the five measurements, all available at $1 \mathrm{kHz}$. The time series show a train of pulses induced in the set-point $\omega^{*}$ of the shaft speed. The deviations in the time series of the other measurements result from the propagation of the set-point disturbance. The order of the measurements in the plot reflects, from top to bottom, the propagation path of the disturbance. This expected propagation path is derived from the model of the system in section 2.3 .

\subsection{Changes in a propagating disturbance}

When a disturbance propagates along a system, its effect on the disturbed system variables changes due to the dynamic characteristics of that system. Table 1 indicates four changes which are commonly observed.

Figure 3 shows a close-up on the measurements of the case study so that these changes can be observed. The start of the disturbance is seen after the $30 \mathrm{~s}$ time instant. The effect of additional time constants is best observed from 


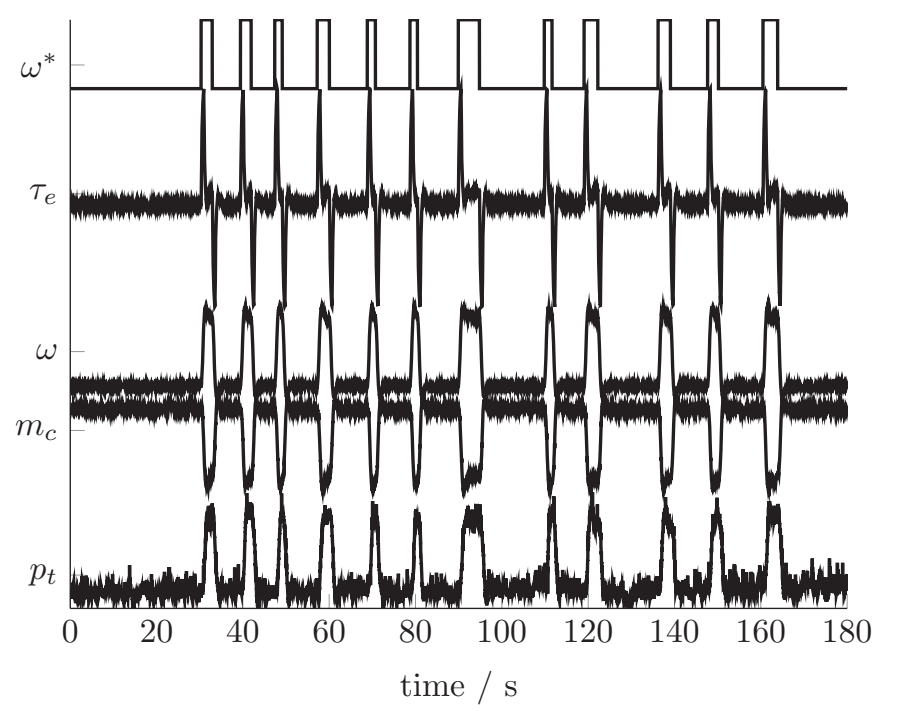

Figure 2: Time series of the original fast-sampled measurements in the case study.

measurement $\omega^{*}$ to $\tau_{e}$, and from $\tau_{e}$ to $\omega$, whereas the effect of dead time is best observed from measurement $\omega$ to $m_{c}$, and from $m_{c}$ to $p_{t}$. Methods which are sensitive to the changes indicated in table 1 can be used to determine which measurements precede others in the propagation path of the disturbance.

\subsection{Propagation path derived from physical models}

The expected propagation path of the set-point disturbance along the measured variables is shown in (1).

$$
\omega^{*} \rightarrow \tau_{e} \rightarrow \omega \rightarrow m_{c} \rightarrow p_{t}
$$

The purpose of this subsection is to justify this expected propagation path from the physical models of the system.

\section{Drive control}

The a.c. voltage-source inverter drive is a power electronics unit capable of controling the rotor speed $\omega$ of induction motors. The electrical drive controls 
Table 1: Common changes in a propagating disturbance due to dynamic characteristics of the system.

\begin{tabular}{ll}
\hline Change & Underlying dynamic characteristic \\
\hline Time lag between the disturbance in & Dead time \\
the measurements of two variables & \\
Low pass filtering, i.e. smoothing of & Time constant \\
the disturbance trend & \\
Decrease in the disturbance & Gain smaller than one \\
magnitude & \\
Addition of noise & Measurement noise or outside \\
& influences \\
\hline
\end{tabular}

directly the electromagnetic torque $\tau_{e}$ output to the induction motor by manipulating the frequency and amplitude of the a.c. voltage $\mathbf{u}_{s}$ output to the motor. The torque set-point $\tau_{e}^{*}$ is given by the outer speed control loop. Therefore, changes in the speed set-point $\omega^{*}$ propagate to the electromagnetic torque $\tau_{e}$.

The speed control loop uses a PID controller based on the rotor speed error $\left(\omega^{*}-\omega\right)$. The torque control algorithm aims at restricting the torque error $\left(\tau_{e}^{*}-\tau_{e}\right)$ within a hysteresis band. The manipulated variables are the switching states $k$ of the inverter, which are selected from an optimum switching table and determine the stator voltage $\mathbf{u}_{s}$ according to

$$
\mathbf{u}_{s}=\frac{2}{3} U_{\text {d.c. }} e^{j(k-1)^{\pi / 3}}
$$

where $U_{\text {d.c. }}$ is the d.c. link voltage. Vas (1998) provides detailed explanation of the drive control algorithms.

\section{Induction motor}

An asynchronous induction motor is formed by a stationary outer part, the stator, which is supplied with an a.c. voltage $\mathbf{u}_{s}$, and a rotating inner part, the 


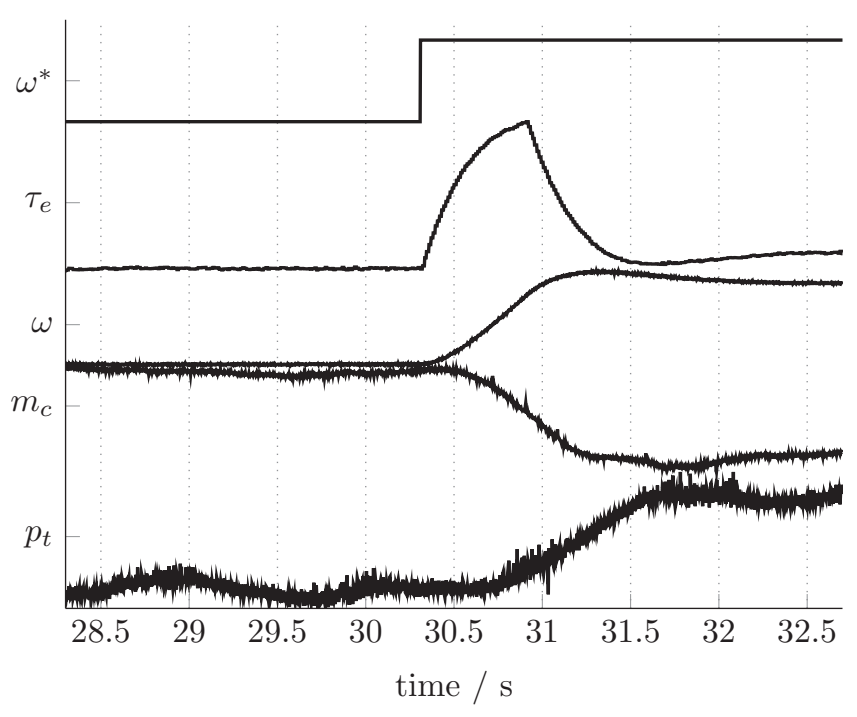

Figure 3: Close-up on the start of a disturbance induced with the set-point $\omega^{*}$. The sequence of plots reflects, from top to bottom, the propagation path of the disturbance.

rotor, which is conductive. The interaction between of the rotating magnetic field of the stator and the rotor current creates a force. The tendency of that force to rotate the rotor is the electromagnetic torque, $\tau_{e}$. Vas (1998) and Holtz (2002) comprehensively explain the mechanism of production of electromagnetic torque in the motor from the frequency and amplitude of the input voltage.

The disturbances in the electromagnetic torque $\tau_{e}$ propagate to the rotor speed $\omega$ according to

$$
\frac{d \omega}{d t}=\frac{1}{J}\left(\tau_{e}-\tau_{L}\right)
$$

which describes the angular momentum balance between the electromagnetic torque, and the torque produced by the load connected to the shaft, $\tau_{L}$. Parameter $J$ is the moment of inertia of the rotating parts.

When the load is a rotating compressor, the load torque $\tau_{L}$ is approximated by Euler's pump equation 


$$
\tau_{L}=\sigma r^{2} \omega\left|m_{c}\right|
$$

which is a function of the fluid mass flow $m_{c}$ through the compressor. Parameter $r$ denotes the compressor impeller radius, and the slip factor $\sigma$ approximates how the fluid moves circumferentially relative to the rotating impeller blades.

\section{Compressor}

The effect of shaft speed $\omega$ on the operation of the compressor is modelled by the compressor characteristic $\Psi_{c}$, which is an adimensional number given by the compressor pressure ratio, $p_{p} / p_{a}$. The compressor characteristic can be considered a steady state nonlinear function of the compressor mass flow $m_{c}$ and shaft speed $\omega$. It is usually approximated by the third order polynomial on the mass flow

$$
\Psi_{c}=c_{0}+c_{1} m_{c}+c_{2} m_{c}^{2}+c_{3} m_{c}^{3}
$$

with the coefficients $c_{i}$ being a second or third order polynomial of the speed $\omega$, as in

$$
c_{i}=c_{i, 0}+c_{i, 1} \omega+c_{i, 2} \omega^{2}+c_{i, 3} \omega^{3}
$$

Changes in the compressor characteristic $\Psi_{c}$ affect the dynamics of the compressor mass flow $m_{c}$ according to

$$
\frac{d m_{c}}{d t}=\frac{A_{c}}{L_{c}}\left(\Psi_{c} \cdot p_{i}-p_{t}\right)
$$

which describes by the momentum balance over the length of the compressor. Parameters $A_{c}$ and $L_{c}$ represent, respectively, the compressor duct cross section and length. Variable $p_{i}$ represents the pressure at the compressor inlet.

The disturbances in the compressor mass flow $m_{c}$ affect the dynamics of the pressure $p_{t}$ in the downstream tank according to the tank mass balance, which can be approximated by 


$$
\frac{d p_{t}}{d t}=\frac{1}{V_{t} \rho_{t} \beta_{T}}\left(m_{c}-m_{t}\right)
$$

The approximation comes from ignoring the rate of temperature change when compared to the rate of pressure change. Parameters $V_{t}, \rho_{t}$ and $\beta_{T}$ represent, respectively, the tank volume, the density of air in the tank, and the compressibility factor of the air at constant temperature.

Variable $m_{t}$ in equation (8) represents the flow through the valve after the tank. This can be considered a steady state function of the tank pressure $p_{t}$ and ambient pressure $p_{a}$ according to

$$
m_{t}=k_{t} \sqrt{p_{t}-p_{a}}
$$

where $k_{t}>0$ is proportional to valve opening.

\section{Non-linear mutual prediction}

\subsection{Self and mutual predictability}

Repeating patterns in a time series can be exploited to predict its future values. A simple example is a purely periodic time series. Once one full cycle is observed, the rest of the time series is known to be a repetition of that same cycle. The top panel in Figure 4 shows another time series, $X$, which has repeating patterns. In this example, the three highlighted segments are all similar, and the figure shows that their future samples, which are marked by crosses, are also similar. This means that if the crossed sample $x_{i+h}$ were unknown, it could be predicted from the other two crossed samples. The prediction of a time series from its own past is known as self-predictability (Kantz and Schreiber, 2003). Applications of this property include removing transient disturbances from oscillatory time series (Cecílio et al., 2015), and testing the non-linearity of a time series (Thornhill, 2005).

Another way to predict sample $x_{i+h}$ is from a second time series, $Y$, which is related to $X$. A relationship between $X$ and $Y$ may occur if one of the time 

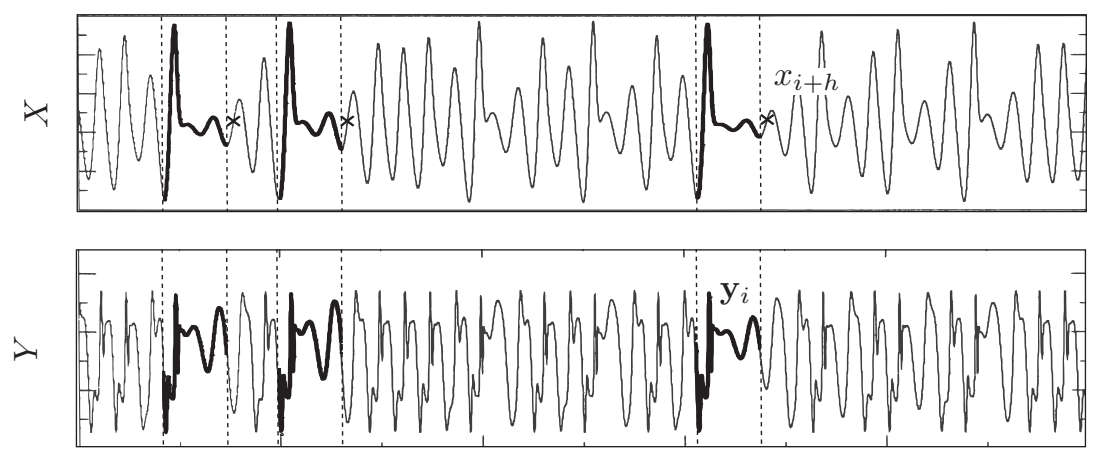

sample number

Figure 4: Illustration of predictability: three identical $X$ samples, marked with crosses, can be indicated by three past identical segments in $X$ (self-predictability) or in $Y$ (non-linear mutual predictability).

series is an input to a system and the other is an output, if both time series are outputs of the same system, or if the systems that generate the time series have a common driver (Schiff et al., 1996).

If time series $X$ and $Y$ are related, then the repetition of a pattern in one of the time series should also imply the repetition of a pattern in the other. This is illustrated in Figure 4. The figure shows that the similar segments highlighted in $X$ correspond to segments in $Y$ which are also similar. This means that the samples similar to $x_{i+h}$ can also be indicated with the aid of time series $Y$. The highlighted segment $\mathbf{y}_{i}$ occurs before sample $x_{i+h}$. The other two highlighted $Y$ segments are significantly similar to $\mathbf{y}_{i}$. Therefore, the $X$ samples which occur $h$ samples after those two highlighted $Y$ segments can be used as predictors for $x_{i+h}$. The prediction of a time series from the past of another is known as non-linear mutual prediction (Schiff et al., 1996; Le Van Quyen et al., 1998). Stockmann et al. (2012) used this property to determine time delays between time series non-linearly correlated.

\subsection{Predictability improvement and directionality}

The prediction of $X$ may be more accurate from the past of $Y$ than from its own past. The same comments can be made about the predictability of time 


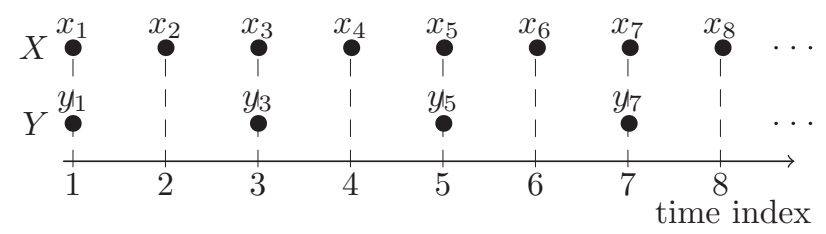

Figure 5: Symbolical time series $X$ and $Y$ with different sampling intervals, with samples represented by dots.

series $Y$ from it own past compared to using the past of $X$. The predictability improvements of $X$ and $Y$ can be compared, and this comparison allows to ascertain whether there is directionality in the relationship between the two time series (Feldmann and Bhattacharya, 2004). If $X$ improves the prediction of $Y$ more than $Y$ improves the prediction of $X$, then one can say that the relationship between the two time series has a direction from $X$ to $Y$.

Bauer et al. (2007b) used predictability improvement to determine the directionality of the propagation of a disturbance between two measurements. The reason is that if $Y$ has propagation features such as time lag, attenuation or added noise in relation to $X$, then these features make $Y$ easier to predict from the past of $X$ than from its own past. The implementation uses nearest neighbors of embedded vectors, which will be detailed in section 4 .

\section{Multi-rate propagation method}

This section explains the adaptation of the uni-rate propagation method by Bauer et al. (2007b) to multi-rate systems. The two symbolical time series represented in Figure 5 are used for illustrating the differences in the formulation of the two methods. The dots in the figure represent the samples. The fast-sampled time series is $X=\left\{\begin{array}{lllllll}x_{1} & x_{2} & x_{3} & x_{4} & x_{5} & \cdots & x_{N_{X}}\end{array}\right\}$, and the slow-sampled is $Y=\left\{\begin{array}{lllll}y_{1} & y_{3} & y_{5} & \cdots & y_{N_{Y}}\end{array}\right\}$. In this example, the sampling interval of the fast-sampled time series, $\Delta^{f}$, is half the sampling interval of the slow-sampled time series, $\Delta^{s}$. 
The objective of the methods is to determine a directionality measure between the two time series.

\subsection{Previous formulation of embedding matrices}

The uni-rate method requires the two time series to be sampled synchronously with the same sampling interval. Therefore, to apply this method to $X$ and $Y$ in Figure 5, samples $\left\{\begin{array}{llll}x_{2} & x_{4} & \cdots\end{array}\right\}$ of the fast-sampled time series need to be discarded to match the slow-sampled time series.

Time series $X$ and $Y$ are then arranged in embedding matrices $\mathbf{X}$ and $\mathbf{Y}$, according to (10a). Each row in $\mathbf{X}$ and $\mathbf{Y}$ is a segment of the time series with $m$ samples. In the example, $m=3$. The rows are known as embedded vectors $\mathbf{x}_{i}$ and $\mathbf{y}_{i}$. The similarity between pairs of embedded vectors will be assessed in a later step of the algorithm. The discarded samples $\left\{\begin{array}{llll}x_{2} & x_{4} & \cdots\end{array}\right\}$ of the fast-sampled time series mean that the embedded vectors $\mathbf{x}_{i}$ are less well characterized, and hence the accuracy of the similarity assessment may be compromised.

$$
\begin{aligned}
& \mathbf{X}=\left[\begin{array}{cccc}
x_{1} & x_{3} & x_{5} \\
x_{3} & x_{5} & x_{7} \\
\vdots & \vdots & \vdots
\end{array}\right] \quad \mathbf{Y}=\left[\begin{array}{ccc}
y_{1} & y_{3} & y_{5} \\
y_{3} & y_{5} & y_{7} \\
\vdots & \vdots & \vdots
\end{array}\right] \quad \mathbf{X}_{h}=\left[\begin{array}{c}
x_{7} \\
x_{9} \\
\vdots
\end{array}\right] \\
& \mathbf{X}=\left[\begin{array}{ccccc}
x_{1} & x_{2} & x_{3} & x_{4} & x_{5} \\
x_{3} & x_{4} & x_{5} & x_{6} & x_{7} \\
\vdots & \vdots & \vdots & \vdots & \vdots
\end{array}\right] \mathbf{Y}=\left[\begin{array}{ccc}
y_{1} & y_{3} & y_{5} \\
y_{3} & y_{5} & y_{7} \\
\vdots & \vdots & \vdots
\end{array}\right] \mathbf{X}_{h}=\left[\begin{array}{ll}
x_{6} & x_{7} \\
x_{8} & x_{9} \\
\vdots &
\end{array}\right]
\end{aligned}
$$

\subsection{New formulation of embedding matrices}

The formulation of the embedding matrix for the slow-sampled time series $Y$ is the same as in section 4.1. The number of samples in each embedded vector $\mathbf{y}_{i}$ is denoted by $m^{s}$. In the example $m^{s}=3$ as illustrated in (10b).

The formulation of the embedding matrix for the fast-sampled time series $X$ is new and avoids the need to discard any samples. The condition imposed 
is that embedded vectors $\mathbf{x}_{i}$ must span the same time duration as embedded vectors $\mathbf{y}_{i}$. As a result, the number of samples $m^{f}$ in each embedded vector $\mathbf{x}_{i}$ is given by

$$
\frac{\left(m^{f}-1\right)}{\left(m^{s}-1\right)}=\frac{\Delta t^{s}}{\Delta t^{f}}
$$

The idea of imposing equal time duration for the embedded vectors instead of equal number $m$ of samples has been used by Cecílio et al. (2014) for multivariate detection in multi-rate systems.

Each embedded vector $\mathbf{x}_{i}$ in the embedding matrix $\mathbf{X}$ must also lag the previous by $\delta^{f}$ samples, where $\delta^{f}$ is given by

$$
\delta^{f}=\frac{\Delta t^{s}}{\Delta t^{f}}
$$

This is to ensure that embedding matrices $\mathbf{X}$ and $\mathbf{Y}$ have the same number of embedded vectors, and that these correspond to the same time intervals./

Equation (10b) illustrates the resulting embedding matrix $\mathbf{X}$ for the running example, for which equations (11) and (12) yield $m^{f}=5$ and $\delta^{f}=2$.

\subsection{Previous formulation of predicted samples}

Array $\mathbf{X}_{h}$ in equation (10a) aligns the predicted samples of $X$ with the corresponding embedded vectors of $\mathbf{X}$ and $\mathbf{Y}$. To assess the self-predictability of $X$, each embedded vector $\mathbf{x}_{i}$ is used in predicting the sample of $X$ that occurs $h$ sampling intervals after the end of the embedded vector. The same sample is also predicted by the embedded vector $\mathbf{y}_{i}$ to yield the mutual predictability of $X$ by $Y$.

The self and mutual predictability of $Y$ are equally formed.

The samples $\left\{\begin{array}{lll}x_{6} & x_{8} & \cdots\end{array}\right\}$ discarded from the fast-sampled time series will not be predicted, and thus will not contribute to the assessment of predictability.

\subsection{New formulation for predicted samples}

The new array $\mathbf{X}_{h}$ is expanded to include the samples which were discarded for the uni-rate method. The expansion is illustrated in equation (10b) with 
the running example. Embedded vectors $\mathbf{x}_{i}$ and $\mathbf{y}_{i}$ now predict not only sample $x_{i+h}$, but also the $\delta^{f}-1$ samples before that.

Array $\mathbf{Y}_{h}$ is formulated as in section 4.3.

\subsection{Similarity measure and nearest neighbors}

The rest of the algorithm follows as in the method by Bauer et al. (2007b).

The Euclidean distance metric is used to assess the similarity between each pair of embedded vectors in one embedding matrix. The purpose is to retrieve, for each embedded vector, the indices of its $k^{\text {th }}$ most similar embedded vectors, which are known as the $k$ nearest neighbors. An example of nearest neighbors is given in Figure 4 by the three highlighted segments of $X$.

For each embedded vector $\mathbf{x}_{i}$, the indices of its $k$ nearest neighbors are denoted $r_{i, j}$, where $j=1 \cdots k$. For each embedded vector $\mathbf{y}_{i}$, the indices of its $k$ nearest neighbors are denoted $s_{i, j}$, where $j=1 \cdots k$.

\subsection{Self-predictability}

To assess the self-predictability of $X$, each row $\mathbf{x}_{i+h}$ in array $\mathbf{X}_{h}$ is compared to its $k$ predictors $\mathbf{x}_{r_{i, j}+h}$. Each of these predictors is the row in $\mathbf{X}_{h}$ corresponding to the embedded vector $\mathbf{x}_{r_{i, j}}$. The comparisons of $\mathbf{x}_{i+h}$ with its predictors are averaged according to

$$
e_{i}\left(\mathbf{x}_{i+h} \mid X\right)=\frac{1}{k} \sum_{j=1}^{k}\left\|\mathbf{x}_{i+h}-\mathbf{x}_{r_{i, j}+h}\right\|
$$

Therefore, quantity $e_{i}\left(\mathbf{x}_{i+h} \mid X\right)$ gives the prediction error of row $\mathbf{x}_{i+h}$ given the past of measurement $X$.

To assess the self-predictability of $Y$, the quantities $e_{i}\left(y_{i+h} \mid Y\right)$ are defined from samples $y_{i+h}$ and its $k$ predictors $y_{s_{i, j}+h}$. Each of these predictors is the sample corresponding to the embedded vector $\mathbf{y}_{s_{i, j}}$.

To illustrate the self-prediction with measurements from the case study, $\omega^{*}$ and $\omega$ are used. In the experimental rig both measurements are sampled at 1 $\mathrm{kHz}$, but in industrial practice $\omega$ could have a slower sampling rate. To represent 
Table 2: Average predictability errors for measurements $\omega^{*}$ and $\omega$.

\begin{tabular}{lll}
\hline self-predictability & $\bar{e}\left(\omega^{*} \mid \omega^{*}\right)$ & $\bar{e}(\omega \mid \omega)$ \\
\cline { 2 - 3 } & 0.0035 & 0.0046 \\
\hline mutual predictability & $\bar{e}\left(\omega^{*} \mid \omega\right)$ & $\bar{e}\left(\omega \mid \omega^{*}\right)$ \\
\cline { 2 - 3 } & 0.0049 & 0.0032 \\
\hline
\end{tabular}

this scenario, $\omega$ is downsampled by a factor of 5000. The errors $e_{i}\left(\mathbf{x}_{i+h} \mid X\right)$ are averaged over $i$ to give the average self-predictability error $\bar{e}(X \mid X)$. The self-predictability error $\bar{e}(Y \mid Y)$ for $Y$ is computed in the same manner. The first row of Table 2 indicates the average self-predictability errors for $\omega^{*}$ and $\omega$.

\subsection{Mutual predictability}

To assess the mutual predictability of $X$ by $Y$, each row $\mathbf{x}_{i+h}$ in array $\mathbf{X}_{h}$ is compared to its $k$ predictors $\mathbf{x}_{s_{i, j}+h}$. Each of these predictors is based on the embedded vector $\mathbf{y}_{s_{i, j}}$. The comparisons of $\mathbf{x}_{i+h}$ with its predictors are averaged according to

$$
e_{i}\left(\mathbf{x}_{i+h} \mid Y\right)=\frac{1}{k} \sum_{j=1}^{k}\left\|\mathbf{x}_{i+h}-\mathbf{x}_{s_{i, j}+h}\right\|
$$

Therefore, quantity $e_{i}\left(\mathbf{x}_{i+h} \mid Y\right)$ gives the prediction error for row $\mathbf{x}_{i+h}$ given the past of measurement $Y$.

To assess the mutual predictability of $Y$ by $X$, the quantities $e_{i}\left(y_{i+h} \mid X\right)$ are defined from samples $y_{i+h}$ and its $k$ predictors $y_{r_{i, j}+h}$. Each of these predictors is now based on the embedded vector $\mathbf{x}_{r_{i, j}}$.

As with self-predictability, the errors $e_{i}\left(\mathbf{x}_{i+h} \mid Y\right)$ and $e_{i}\left(y_{i+h} \mid X\right)$ are averaged over all samples to give the average mutual predictability errors $\bar{e}(X \mid Y)$ and $\bar{e}(Y \mid X)$. The last row of Table 2 indicates the average mutual predictability errors for $\omega^{*}$ and $\omega$. 
The prediction error increases from $\bar{e}\left(\omega^{*} \mid \omega^{*}\right)$ to $\bar{e}\left(\omega^{*} \mid \omega\right)$ which shows that the prediction of $\omega^{*}$ is less accurate from the past of $\omega$ than from its own past. Conversely, comparing $\bar{e}(\omega \mid \omega)$ with $\bar{e}\left(\omega \mid \omega^{*}\right)$ shows that the past of $\omega^{*}$ improves the prediction of $\omega$. This difference has a meaning, which is explained in the next subsection.

\subsection{Predictability improvement and directionality}

The predictability improvement measure $H(X \mid Y)$ is defined as

$$
H(X \mid Y)=\frac{\bar{e}(X \mid Y)}{\bar{e}(X \mid X)}
$$

which compares the prediction of $X$ from the past of $Y$ with the prediction of $X$ from its own past. The complementary $H(Y \mid X)$ is defined analogously.

The predictability improvements of $X$ and $Y$ are compared using

$$
H_{X \rightarrow Y}=H(X \mid Y)-H(Y \mid X)
$$

Equation (16) yields $H_{X \rightarrow Y}$, a measure of the directionality of the influence between $X$ and $Y$. If $H_{X \rightarrow Y}$ is positive the influence is directed from $X$ to $Y$, if $H_{X \rightarrow Y}$ is negative the influence is directed from $Y$ to $X$.

The directionality measure for measurements $\omega^{*}$ and $\omega$ is $H_{\omega^{*} \rightarrow \omega}=0.71$. This value is positive, which indicates that the disturbance affecting these two measurements propagated from $\omega^{*}$ to $\omega$. This result agrees with the expected propagation path, as discussed in section 2.3.

\section{Results with the uni-rate and multi-rate methods}

This section applies the uni-rate method by Bauer et al. (2007b) and the proposed multi-rate method to the same four pairs of measurements from the case study. The objective is to evaluate the improvement given by the multi-rate method in case of a multi-rate data set.

As mentioned before, all measurements in the case study were sampled at 1 kHz. By downsampling selected measurements, this fast-sampled data set was used to carry the four tests listed below. 


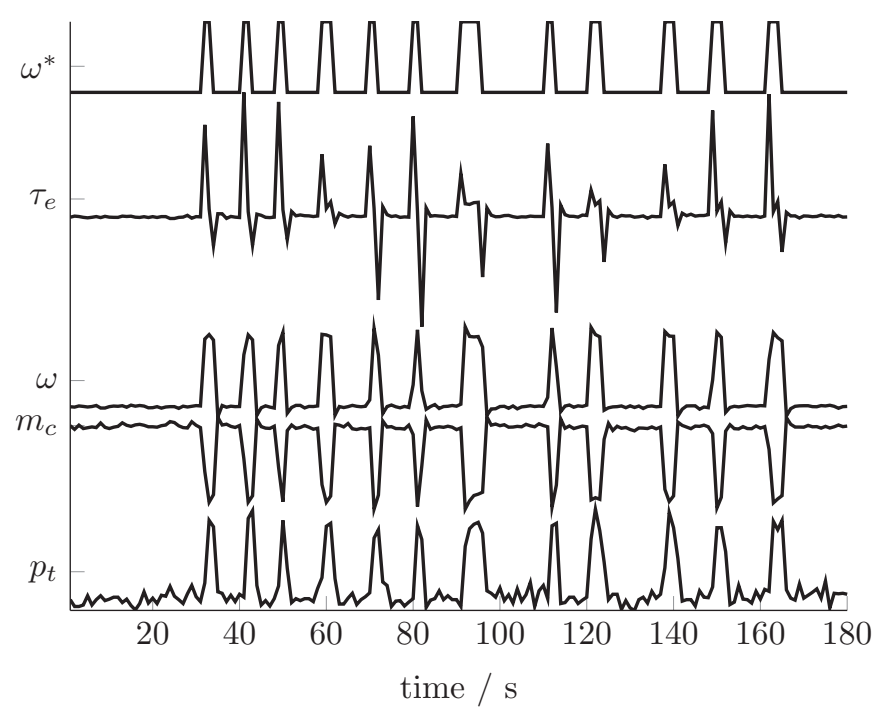

Figure 6: Measurements from the reference case study after downsampling by a factor of 5000.

1. The original fast-sampled measurements are analysed with the uni-rate method. This test is the benchmark.

2. All measurements are downsampled by a factor of 5000, resulting in the trends shown in Figure 6. The measurements are analysed with the unirate method. This test represents the case of a multi-rate data set where available fast measurements have to be downsampled to allow the uni-rate analysis.

3. For each pair of measurements, the measurement known to precede in the propagation path keeps the original fast sampling rate and the measurement following is downsampled by a factor of 5000. The multi-rate method is used. This test represents a case of a multi-rate data set where the multi-rate method allows to use of all the data from the fast-sampled measurement.

4. For each pair of measurements, the measurement known to precede in the propagation path is downsampled by a factor of 5000 and the measurement following keeps the original fast sampling rate. The multi-rate method is used. This test is used to check that the precedence order predicted by 
Table 3: Directionality measures $H_{X \rightarrow Y}$ obtained with the case study in four tests. Symbols $F$ and $S$ indicate whether variables $X$ and $Y$ have the fast or slow sampling rate.

\begin{tabular}{lllll}
\hline & \multicolumn{2}{c}{ Uni-rate method } & \multicolumn{2}{c}{ Multi-rate method } \\
\cline { 2 - 5 } & Test 1 & Test 2 & Test 3 & Test 4 \\
& $1: F \rightarrow F$ & $2: S \rightarrow S$ & $3: F \rightarrow S$ & $4: S \rightarrow F$ \\
\hline$H_{\omega^{*} \rightarrow \tau_{e}}$ & 0.82 & 0.68 & 0.66 & 0.50 \\
$H_{\tau_{e} \rightarrow \omega}$ & 0.52 & 0.03 & 0.25 & 0.35 \\
$H_{\omega \rightarrow m_{c}}$ & 0.96 & 0.46 & 0.71 & 0.62 \\
$H_{m_{c} \rightarrow p_{t}}$ & 1.90 & 0.81 & 0.73 & 0.81 \\
\hline
\end{tabular}

the method is not influenced by which measurements have the fast and slow sampling rates.

Table 3 shows the directionality measures $H_{X \rightarrow Y}$ in the four tests (numbered columns) for the four pairs of measurements (rows). Symbols $F$ and $S$ indicate whether variables $X$ and $Y$ have the fast or slow sampling rate. The expected result is that all selected pairs have positive $H_{X \rightarrow Y}$ measures. The reason is that the model in section 2.3 was consulted so that the measurements chosen as $X$ precede the measurements chosen as $Y$ in the propagation path.

The first observation is that all $H_{X \rightarrow Y}$ measures are indeed positive, which means that all tests correctly recognized the direction of propagation of the disturbance. As expected, the strongest directionality indications are yielded when all measurements have the fast sampling rate. Test 2 shows that using the uni-rate method with slow-sampled measurements detects the directionality, but much less strongly. The results of tests 3 and 4 show that the new multi-rate method gives some benefit in this case study. The comment is justified on the basis that some of the directionality indicators are larger when the fast measurements are included without downsampling.

It is noted that some of the directionality indicators do not increase from 
test 2 to tests 3 and 4 . This is because, in this case study, the duration of the disturbance in the fast-sampled measurements is long enough to be captured by slow sampling rate. These results suggest that a pragmatic approach to detecting directionality in multi-rate data sets is to start the analysis with a uni-rate analysis on downsampled data at the slow sampling rate, because this analysis is less computationally intensive. If the results are ambiguous, then the multi-rate method may be applied selectively to strengthen confidence in the results. For instance, the uni-rate result of 0.03 in $H_{\tau_{e} \rightarrow \omega}$ would need to be investigated more thoroughly by means of a multi-rate analysis to confirm the directionality. Ambiguities such as this are more likely in data sets in which the duration of the disturbance is shorter in the fast-sampled electromechanical measurements than in the slow-sampled process measurements. In these cases, the slower sampling rate may be enough to capture the disturbance in the process measurements but not in the electromechanical measurements.

\section{Conclusions}

The work in this paper contributes to the extension of process monitoring and diagnosis to the electrical and mechanical utilities, which has been highlighted as a need by industrial commentators. The paper proposes a multi-rate method to identify the propagation path of a persistent disturbance, and shows that it is possible to track disturbances passing between electrical, mechanical, and process systems. The motivation to extract the propagation path of a disturbance from measurement data is that it allows to diagnose the root cause of the disturbance without the need of a model. To that end, the signal analysis method is sensitive to features that arise in the data when the disturbance propagates along a system, namely time lags, low pass filtering, decrease in magnitude, and added noise. The motivation for a multi-rate method is that measurements from process and electromechanical systems often have different sampling rates. The challenge with different sampling rates is that downsampling the fast-sampled measurement to the slower rate may cause loss 
of information, while interpolating the slow-sampled measurements to the faster rate may create false information. The multi-rate method uses the information available.

The proposed multi-rate method adapts the method by Bauer et al. (2007b) to determine the propagation path of a persistent disturbance. The original method is only applicable to measurements with the same sampling rate. The new method was tested on a multi-rate data set derived from an experimental case study, in which the root cause was known and the propagation path was derived from first principles. In the same multi-rate data set, the uni-rate method required the fast measurements to be downsampled to the slower rate. The multi-rate method determined the correct propagation path, and for some pairs of measurements yielded better results than the uni-rate method.

The improvement was not always dramatic. This suggested that a pragmatic approach to detecting directionality in multi-rate data sets is to start the analysis with a uni-rate analysis on data downsampled to the slow sampling rate, and apply the multi-rate method selectively when the initial results are ambiguous. Ambiguity is likely when the duration of the disturbance is shorter in the fast-sampled electromechanical measurements than in the slow-sampled process measurements. For those data sets, the method proposed in this paper is a promising direction for research. Future studies should explore the conditions under which the multi-rate method yields better results than the uni-rate method, including conditions on the system dynamics and the periodicity of the disturbance. This study could be done using an array of simulated case studies.

\section{Acknowledgements}

The authors gratefully acknowledge the financial support from the Portuguese Foundation for Science and Technology (FCT) under Fellowship SFRH/BD/61384/2009 and the Marie Curie FP7-IAPP project "REAL-SMART - Using real-time measurements for monitoring and management of power transmission dynamics for the Smart Grid", Contract No: PIAP-GA-2009-251304. 
Bauer, M., Cox, J. W., Caveness, M. H., Downs, J. J., Thornhill, N. F., 2007a. Finding the direction of disturbance propagation in a chemical process using transfer entropy. IEEE Transactions on Control Systems Technology 15 (1), $12-21$.

Bauer, M., Cox, J. W., Caveness, M. H., Downs, J. J., Thornhill, N. F., 2007b. Nearest neighbors methods for root cause analysis of plantwide disturbances. Industrial \& Engineering Chemistry Research 46 (18), 5977-5984.

Cecílio, I. M., Chen, S.-L., Thornhill, N. F., 2011. Importance of auxiliary systems for process fault detection and diagnosis. In: Proceedings of the 19th Mediterranean Conference on Control \& Automation. IEEE, pp. 952-957.

Cecílio, I. M., Ottewill, J. R., Fretheim, H., Thornhill, N. F., 2014. Multivariate detection of transient disturbances for uni- and multirate systems. IEEE Transactions on Control System Technology PP (99), 1.

Cecílio, I. M., Ottewill, J. R., Fretheim, H., Thornhill, N. F., 2015. Removal of transient disturbances from oscillating measurements using nearest neighbors imputation. Journal of Process Control[Preprint].

Cecílio, I. M., Ottewill, J. R., Pretlove, J., Thornhill, N. F., 2014. Nearest neighbors method for detecting transient disturbances in process and electromechanical systems. Journal of Process Control 24 (9), 1382-1393.

Feldmann, U., Bhattacharya, J., 2004. Predictability improvement as an asymmetrical measure of interdependence in bivariate time series. International Journal of Bifurcation and Chaos 14 (2), 505-514.

Holtz, J., 2002. Sensorless control of induction motor drives. Proceedings of the IEEE 90 (8), 1359-1394.

Horch, A., Cox, J., Bonavita, N., 2007. Peak performance - Root cause analysis of plant-wide disturbances. ABB Review 1, 24-29. 
Kantz, H., Schreiber, T., 2003. Nonlinear Time Series Analysis. Cambridge University Press.

Le Van Quyen, M., Adam, C., Baulac, M., Martinerie, J., Varela, F. J., 1998. Nonlinear interdependencies of EEG signals in human intracranially recorded temporal lobe seizures. Brain Research 792 (1), 24-40.

Lindholm, A., Carlsson, H., Johnsson, C., 2011. A general method for handling disturbances on utilities in the process industry. In: Proceedings of the 18th IFAC World Congress. IFAC, Milano, pp. 952-957.

Naghoosi, E., Huang, B., Domlan, E., Kadali, R., 2013. Information transfer methods in causality analysis of process variables with an industrial application. Journal of Process Control 23 (9), 1296-1305.

Reeves, T., 2005. Optimising process equipment performance. Petroleum Technology Quarterly $10(4), 93-97$.

Schiff, S. J., So, P., Chang, T., Burke, R. E., Sauer, T., 1996. Detecting dynamical interdependence and generalized synchrony through mutual prediction in a neural ensemble. Physical Review E 54 (6), 6708.

Schiltz, R., 2008. Condition monitoring under control. Plant Services 29 (9), 39 -42 .

Stockmann, M., Haber, R., Schmitz, U., 2012. Source identification of plantwide faults based on $k$ nearest neighbor time delay estimation. Journal of Process Control 22 (3), 583-598.

Thornhill, N. F., 2005. Finding the source of nonlinearity in a process with plant-wide oscillation. IEEE Transactions on Control Systems Technology $13(3), 434-443$.

Thornhill, N. F., Horch, A., 2007. Advances and new directions in plant-wide disturbance detection and diagnosis. Control Engineering Practice 15 (10), 1196. 
Vas, P., 1998. Sensorless vector and direct torque control. Oxford University Press, Oxford New York.

Yang, F., Xiao, D., 2012. Progress in root cause and fault propagation analysis of large-scale industrial processes. Journal of Control Science and Engineering, 10. 